\title{
Chemomechanical Coupling of Molecular Motors: Thermodynamics, Network Representations, and Balance Conditions
}

\author{
Reinhard Lipowsky • Steffen Liepelt
}

Published online: 8 May 2009

(C) Springer Science+Business Media, LLC 2009

\section{Erratum to: J Stat Phys (2008) 130: 39-67 DOI 10.1007/s10955-007-9425-7}

The derivation of (6.15) contains an implicit assumption that restricts its general validity and led to a wrong conclusion about the two relations (9.3) and (9.4).

As explained in Sect. 4.1, each motor state $i$ corresponds to an ensemble of substates, which is characterized by the Helmholtz free energy $H_{i}$ and the internal energy $U_{i}$, see (4.2) and (4.3). These two quantities define the entropy $S_{i}=\left(U_{i}-H_{i}\right) / T$ of state $i$ at temperature $T$. During a transition $|i j\rangle$ from state $i$ to state $j$, the motor produces, on average, the entropy $\Delta S_{i j}=k_{B} \ln \left(\omega_{i j} / \omega_{j i}\right)$, see (6.13). We then identified $T \Delta S_{i j}$ with the average heat $Q_{i j}$ that the motor releases during the transition $|i j\rangle$ into the heat reservoir. The latter identification is, however, restricted to motor states $i$ and $j$ with $S_{i}=S_{j}$.

For the more general case with $S_{i} \neq S_{j}$, the average produced entropy during the transition $|i j\rangle$ satisfies the relation

$$
\Delta S_{i j}=k_{B} \ln \left(\omega_{i j} / \omega_{j i}\right)=S_{j}-S_{i}+Q_{i j} / T
$$

where $S_{j}-S_{i}$ and $Q_{i j} / T$ represent the entropy change of the motor molecule and of the heat bath, respectively. The entropy differences $S_{j}-S_{i}$ cancel out from the entropies $\Delta S\left(\mathcal{C}_{v}^{d}\right)$ for the dicycles $\mathcal{C}_{v}^{d}$, see (6.8), as well as from the entropy production rates (6.7) and (6.6). Indeed, the general expression (6.6) for the entropy production rate in the steady state remains unchanged if we substitute $\ln \left(\omega_{i j} / \omega_{j i}\right)$ by $\ln \left(\omega_{i j} / \omega_{j i}\right)+A_{j}-A_{i}$ with any state function $A_{i}$ as follows from flux conservation at each state (or vertex) $i$. Therefore, the decomposition (1) is not directly imposed by the form of (6.6) but is consistent with the corresponding

The online version of the original article can be found under doi:10.1007/s10955-007-9425-7.

R. Lipowsky $(\bowtie) \cdot S$. Liepelt

Max-Planck-Institute of Colloids and Interfaces, Science Park Golm, 14424 Potsdam, Germany

e-mail: Reinhard.Lipowsky@ mpikg.mpg.de 
decomposition of macroscopic systems [1] and can be checked directly for a system consisting of only two states $i$ and $j$ not coupled to any other reservoir apart from the heat bath.

When the relation (1) is inserted into the local energy balance relation (4.4), one obtains the free energy differences

$$
H_{j}-H_{i}=U_{j}-U_{i}-T\left(S_{j}-S_{i}\right)=\Delta \mu_{i j}-\ell_{i j} F-k_{B} T \ln \left(\omega_{i j} / \omega_{j i}\right),
$$

which generalizes (6.15) to the case $S_{i} \neq S_{j}$.

All relations of our article that follow after equation (6.15) and involve the internal energies can now be generalized by simply replacing the internal energies $U_{i}$ by the free energies $H_{i}$. When this substitution is made in equations (8.1)-(8.4), for example, one obtains explicit expressions for the landscape of the free energies $H_{i}$. Likewise, the constrained equilibrium relation (9.3) now attains the more general form

$$
\frac{\omega_{b a}}{\omega_{a b}}=\frac{\exp \left[-H_{a} / k_{B} T\right]}{\exp \left[-H_{b} / k_{B} T\right]} e^{\left(-\Delta \mu_{a b}+\ell_{a b} F\right) / k_{B} T} \equiv \frac{P_{a}^{\mathrm{ce}}}{P_{b}^{\mathrm{ce}}} .
$$

For $F=0$, i.e., in the absence of a work reservoir, (3) reduces to relation (9.4) as obtained by Hill and Simmons [2].

We thank Thomas Weikl for stimulating discussions about protein folding.

\section{References}

1. Callen, H.B.: Thermodynamics and an Introduction to Thermostatistics. Wiley, New York (1985)

2. Hill, T.L., Simmons, R.M.: Free energy levels and entropy production associated with biochemical kinetic diagrams. Proc. Nat. Acad. Sci. U.S.A. 73, 95-99 (1976) 